\title{
Two Types of Numerical Approximate Solutions for Clairaut's Equation
}

\author{
Khawla A.Al-Zubaidy \\ University of Baghdad, College of Engineering, Department of Mechanic Engineering
}

\begin{abstract}
The main purpose of this work is to find two types of numerical approximate solutions for Clairaut equation by method of moment and Galerkin method and determination which one is the best.
\end{abstract}

Keywords: Numerical approximate solution, Clairaut equation, first order differential equation, method of moment, Galerkin method.

\section{Introduction}

Alexis Claude Clairaut is French mathematician born in Parisian (1713) and died in1765, [1].

This paper consists of three sections; the formula and the solution for the Clairaut equation are illustrated in the first section. While the second section presented the methods for find the numerical approximate solution which are the moment and Galerkin methods and the third section considered the numerical examples to clarify these methods and finally in the last section the compare between these methods for which gives best numerical approximate solution near to the general solution.

\section{The Formula and the Solution Method for the Clairaut Equation [2], [3]}

Clairaut's equation is a first order differential equation has the form

$$
\mathrm{n}=\mathrm{sn}^{\prime}+\kappa n^{\prime}
$$

Where $\kappa$ is a suitable function.

The solution of this equation can be obtained by letting $\mathrm{n}^{\prime}=$ $h(s)$, so that

$$
\mathrm{n}=\mathrm{sh}+\kappa \mathrm{h}
$$

By differentiating each side with respect to s, we get

$$
\begin{aligned}
& n^{\prime}=s^{\prime}+h+\frac{d \kappa}{d h} h^{\prime} \\
& h=h^{\prime}+h+\frac{d \kappa}{d h} h^{\prime}
\end{aligned}
$$

$$
\left(\mathrm{s}+\frac{\mathrm{d} \kappa}{\mathrm{dh}}\right) \mathrm{h}^{\prime}=0
$$

So we obtain two possible solutions for (3) which are

$h^{\prime}=0 \quad$ or $\quad \mathrm{s}+\frac{\mathrm{d} \kappa}{\mathrm{dh}}=0$

For the first case

$\mathrm{h}^{\prime}=\frac{\mathrm{dh}}{\mathrm{ds}}=0$

By integrate each side with respect to $\mathrm{s}$, we obtain $\mathrm{h}=\mathrm{b}$, where $\mathrm{c}$ is constant
Then from equation (2) we get the general solution

$$
\mathrm{n}=\mathrm{bs}+\kappa(\mathrm{b})
$$

When we applying an initial condition $\mathrm{n}\left(\mathrm{s}_{0}\right)=\mathrm{n}_{0}$, we get the regular solution

For the second case

$\mathrm{s}+\frac{\mathrm{d} \kappa}{\mathrm{dh}}=0$

Simplified that, we get

$\mathrm{s}=-\frac{\mathrm{d} \kappa}{\mathrm{dh}}$

This equation along with the rearranged equation (2)

$\mathrm{n}=-\mathrm{h} \frac{\mathrm{d} \kappa}{\mathrm{dh}}+\kappa(\mathrm{h})$

Which is singular solution.

\section{Methods for Find Numerical Approximate Solution [4], [5]}

Suppose $\tilde{\mathrm{n}}(\mathrm{s})$ is the numerical approximated solution as the following form

$$
\tilde{\mathrm{n}}(\mathrm{s}) \approx \sum_{\alpha=1}^{\mathrm{M}} \zeta_{\alpha} \psi_{\alpha}(\mathrm{s})+\psi_{0}(\mathrm{~s})
$$

Where $\psi_{0}(\mathrm{~s})$ satisfy the initial conditions.

Substitution this form into given Clairaut's equation we get the residual of the numerical approximation solution notion by $\mathrm{N}(\mathrm{s})$.

In both methods in this work put

$$
\int_{\Omega} \mathrm{N}(\mathrm{s}) \mathrm{L}_{\alpha} \mathrm{ds}=0 \quad(\alpha=1,2, \ldots, \mathrm{M})
$$

where the number of the weighted $\mathrm{L}$ is exactly equal the number of $\zeta_{\alpha}$ in $\tilde{\mathrm{n}}(\mathrm{s})$.

\subsection{Method of Moment [6], [7]}

In this method $\mathrm{L}_{\alpha}$ are chosen from the family of polynomials, that is mean

$$
\mathrm{L}_{\alpha}=\mathrm{s}^{\alpha} \quad(\alpha=1,2, \ldots, \mathrm{M})
$$

3.2 Galerkin Method [8], [9]

In Galerkin method $\mathrm{L}_{\alpha}$ are considered as 


\section{International Journal of Science and Research (IJSR) \\ ISSN (Online): 2319-7064}

Index Copernicus Value (2015): 78.96 | Impact Factor (2015): 6.391

$$
\mathrm{L}_{\alpha}=\psi_{\alpha} \quad(\alpha=1,2, \ldots, \mathrm{M})
$$

\section{Application Examples}

\section{Example (1):}

Take the equation $n=s n^{\prime}+(n)^{3}, n(0)=n_{0}$.

\section{Solution:}

To find the general solution

Put $h=n^{\prime}$. Then $\kappa(h)=h^{3}$

$\frac{\mathrm{d} \kappa}{\mathrm{dh}}=3 \mathrm{~h}^{2}$

$\mathrm{n}_{0}=\mathrm{b}(0)+\mathrm{b}^{3} \Rightarrow \mathrm{b}=\mathrm{n}_{0}^{\frac{1}{3}}$

Hence the general solution is $\mathrm{n}=\mathrm{n}_{0}^{\frac{1}{3}} \mathrm{~s}+\mathrm{n}_{0}$

To find the singular solution $\mathrm{n}=-2 \mathrm{~h}^{3}, \mathrm{~s}=-3 \mathrm{~h}^{2}$

So $n=\mp 2\left(\frac{-s}{3}\right)^{\frac{3}{2}}$ is the singular solution.

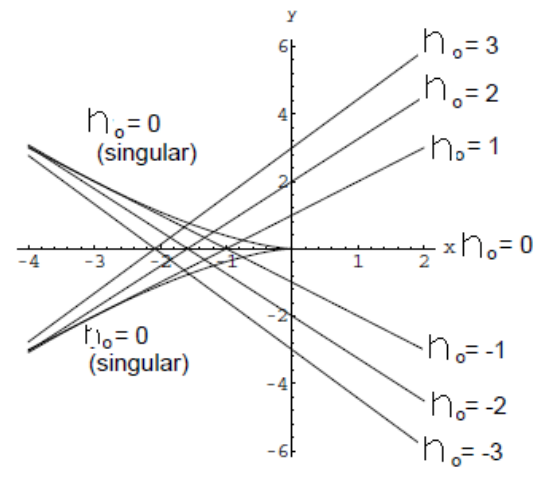

Figure 1: The singular and general solutions

To find the numerical approximate solution

We have $\mathrm{n}=\mathrm{sn}^{\prime}+(\mathrm{n})^{3}, \mathrm{n}(0)=1$ and $\mathrm{n}(1)=0$.

$\psi_{0}(s)=\mathrm{j}+\mathrm{vs}$ which is satisfy the initial condition.

So $\psi_{0}(\mathrm{~s})=1-\mathrm{s}$.

Put $\psi_{1}(\mathrm{~s})=\mathrm{s}, \quad \psi_{1}(\mathrm{~s})=\mathrm{s}^{2}$.

Thus $\tilde{\mathrm{n}}(\mathrm{s}) \approx \zeta_{1} \psi_{1}(\mathrm{~s})+\zeta_{2} \psi_{2}(\mathrm{~s})+\psi_{0}(\mathrm{~s})=1+\left(\zeta_{1}-1\right) \mathrm{s}+\zeta_{2} \mathrm{~s}^{2}$

After substitute $\frac{\partial \tilde{\mathrm{n}}}{\partial \mathrm{s}}=\zeta_{1}-1+2 \mathrm{~s} \zeta_{2} \quad, \quad \frac{\partial^{2} \tilde{\mathrm{n}}}{\partial \mathrm{s}^{2}}=2 \zeta_{2} \quad, \quad \frac{\partial^{3} \tilde{\mathrm{n}}}{\partial \mathrm{s}^{3}}=0$ in given Clairaut equation we get that

$\mathrm{N}(\mathrm{s})=\zeta_{1} \psi_{1}(\mathrm{~s})+\zeta_{2} \psi_{3}(\mathrm{~s})+\psi_{0}(\mathrm{~s})=1+\zeta_{1} \mathrm{~s}-\zeta_{2} \mathrm{~s}^{2}$

By using the method of moment

$\mathrm{L}_{1}=\mathrm{s}^{0}=1$ and $\mathrm{L}_{2}=\mathrm{s}^{1}$

By solving $\int_{0}^{1} \mathrm{~N}(\mathrm{~s}) \mathrm{L}_{\alpha} \mathrm{ds}=0 \quad(\alpha=1,2)$ we obtain a system after solving it we get $\zeta_{1}=1.890$ and $\zeta_{2}=0$.

Thus $\tilde{\mathrm{n}}_{1}(\mathrm{~s})=1+0.890 \mathrm{~s}$.

By solving $\int_{1}^{2} \mathrm{~N}(\mathrm{~s}) \mathrm{L}_{\alpha} \mathrm{ds}=0 \quad(\alpha=1,2)$ we obtain a system after solving it we get $\zeta_{1}=1.5$ and $\zeta_{2}=0.00001 \approx 0$.

Thus $\tilde{\mathrm{n}}_{1}(\mathrm{~s})=1+0.5 \mathrm{~s}$.
Hence if we take the integration on any interval $[a, b]$ we acquire linear equation.

By using the Galerkin method

$\mathrm{L}_{1}=\mathrm{s}$ and $\mathrm{L}_{2}=\mathrm{s}^{2}$

By solving $\int_{0}^{1} \mathrm{~N}(\mathrm{~s}) \mathrm{L}_{\alpha} \mathrm{ds}=0 \quad(\alpha=1,2)$ we obtain a system after solving it we get $\zeta_{1}=2.009$ and $\zeta_{2}=1.099$.

Thus $\tilde{\mathrm{n}}_{2}(\mathrm{~s})=1+1.009 \mathrm{~s}+1.099 \mathrm{~s}^{2}$

By solving $\int_{1}^{2} \mathrm{~N}(\mathrm{~s}) \mathrm{L}_{\alpha} \mathrm{ds}=0 \quad(\alpha=1,2)$ we obtain a system after solving it we get $\zeta_{1}=4.135$ and $\zeta_{2}=1.889$.

Thus $\tilde{\mathrm{n}}_{2}(\mathrm{~s})=1+3.135 \mathrm{~s}+1.889 \mathrm{~s}^{2}$

Therefore if we take the integration on any interval [a,b] we do not acquire linear equation.

Example (2):

Take the equation $\mathrm{n}=\mathrm{sn}^{\prime}+\left(\mathrm{n}^{\prime}\right)^{2}, \mathrm{n}(0)=1$ and $\mathrm{n}(1)=0$.

Solution:

To find the general solution

Put $\mathrm{h}=\mathrm{n}^{\prime}$. Then $\mathrm{n}=\mathrm{s} \mathrm{h}+2 \mathrm{~h}^{2}$

$\mathrm{n}^{\prime}=\mathrm{sh}^{\prime}+\mathrm{h}+4 \mathrm{hh}^{\prime} \Rightarrow \mathrm{h}^{\prime}(\mathrm{s}+4 \mathrm{~h})=0$

$\mathrm{h}^{\prime}=0$ or $\mathrm{s}+4 \mathrm{~h}=0$

$\mathrm{h}^{\prime}=0 \Rightarrow \mathrm{h}=\xi$

Hence the general solution is

$\mathrm{n}=\xi \mathrm{s}+2 \xi^{2}$

To find the singular solution

$\mathrm{s}+4 \mathrm{~h}=0 \Rightarrow \mathrm{h}=\frac{-\mathrm{s}}{4}$

So $\mathrm{n}=\frac{-1}{8} \mathrm{~s}^{2}$ is the singular solution.

To find the numerical approximate solution

$\psi_{0}(s)=\mathrm{j}+\mathrm{vs}$ which is satisfy the initial condition. So $\psi_{0}(\mathrm{~s})=1-\mathrm{s}$.

Put $\quad \psi_{1}(\mathrm{~s})=\mathrm{s}+\mathrm{s}^{2}, \psi_{1}(\mathrm{~s})=\mathrm{s}^{2}$.

Thus $\tilde{\mathrm{n}}(\mathrm{s})=1+\zeta_{1} \mathrm{~s}+\left(\zeta_{2}-\zeta_{1}\right) \mathrm{s}^{2}$

Therefore

$\mathrm{N}(\mathrm{s})=\zeta_{1} \psi_{1}(\mathrm{~s})+\zeta_{2} \psi_{3}(\mathrm{~s})+\psi_{0}(\mathrm{~s})=1+\zeta_{1} \mathrm{~s}-\zeta_{2} \mathrm{~s}^{2}$

By using the method of moment

$\mathrm{L}_{1}=\mathrm{s}^{0}=1$ and $\mathrm{L}_{2}=\mathrm{s}^{1}$

By solving $\int_{0}^{1} \mathrm{~N}(\mathrm{~s}) \mathrm{L}_{\alpha} \mathrm{ds}=0 \quad(\alpha=1,2)$ we obtain a system after solving it we get $\zeta_{1}=0$ and $\zeta_{2}=0.999$.

Thus $\tilde{\mathrm{n}}_{1}(\mathrm{~s})=1+0.999 \mathrm{~s}^{2}$.

By solving $\int_{1}^{2} \mathrm{~N}(\mathrm{~s}) \mathrm{L}_{\alpha} \mathrm{ds}=0 \quad(\alpha=1,2)$ we obtain a system after solving it we get $\zeta_{1}=0.000001 \approx 0$ and $\zeta_{2}=1.899$.

Thus $\tilde{\mathrm{n}}_{1}(\mathrm{~s})=1+1.899 \mathrm{~s}$.

Hence if we take the integration on any interval $[a, b]$ we acquire linear equation.

By using the Galerkin method

$\mathrm{L}_{1}=\mathrm{s}$ and $\mathrm{L}_{2}=\mathrm{s}^{2}$ 
By solving $\int_{0}^{1} \mathrm{~N}(\mathrm{~s}) \mathrm{L}_{\alpha} \mathrm{ds}=0 \quad(\alpha=1,2)$ we obtain a system after solving it we get $\zeta_{1}=0.099$ and $\zeta_{2}=1.099$.

Thus $\tilde{\mathrm{n}}_{2}(\mathrm{~s})=1+0.099 \mathrm{~s}+\mathrm{s}^{2}$

By solving $\int_{1}^{2} \mathrm{~N}(\mathrm{~s}) \mathrm{L}_{\alpha} \mathrm{ds}=0 \quad(\alpha=1,2)$ we obtain a system after solving it we get $\zeta_{1}=1.001$ and $\zeta_{2}=3.221$.

Thus $\tilde{\mathrm{n}}_{2}(\mathrm{~s})=1+1.001 \mathrm{~s}+2.22 \mathrm{~s}^{2}$

Hence if we take the integration on any interval $[a, b]$ we do not acquire linear equation.

\section{Conclusion}

The numerical approximate solution gives by method of moment is the best with respect to the Galerkin method since the first method represent the numerical approximate solution as lines which is exactly the solution for Clairaut's equation, while the second method not gives that.

\section{References}

[1] J. Casey, "Clairaut's Hydrostatics: A Study in Contrast," American Journal of Physics, Vol. 60, pp.549-554, 1992.

[2] M. Joseph Powers, "Lecture Notes on Mathematical Methods," Notre Dame, Indiana, USA, 2012.

[3] I. M. Gelfand and S. V. Fomin "Calculus of Variations," Dover, New York, NY, 2000.

[4] J.N. Reddy, "An introduction to the finite element Method," Third Edition, McGraw-Hill Series in Mechanical Engineering, 2006.

[5] J.N. Reddy, "Energy Principles and Variational Methods in Applies Mechanics," $2^{\text {nd }}$ ed., John Wiley, New York, 2002.

[6] P.M. Gresho and R.L. Sani, "Incompressible Flow and the Finite Element Method," John Wiley, West Sussex, UK, 2000.

[7] P. V. O’Neil "Advanced Engineering Mathematics," Sixth Edition, CL-Engineering, NY, 2006.

[8] J.N. Reddy, and D.K. Gartling "The Finite Element Method in Heat Transfer and Fluid Dynamic," $2^{\text {nd }}$ ed. CRC Press, Boca Raton, FL, 2001.

[9] E. Kreyszig, "Advanced Engineering Mathematics," Ninth Edition, Wiley, New York, NY, 2005. 